\section{OP29 THE LONG-TERM TRAJECTORIES OF COGNITIVE AND MENTAL HEALTH OF UK PENSIONERS: THE IMPACT OF WORK STATUS AND CHOICES}

${ }^{1}$ Baowen Xue*, ${ }^{2}$ Manacy Pai, 'Minhao Luo. 'Department of Epidemiology and Public Health, UCL, London, UK; ${ }^{2}$ Department of Sociology, Kent State University, Kent, USA

\subsection{6/jech-2021-SSMabstracts.29}

Background At a time when older adults are encouraged to work through later life, far less is known about why some individuals work beyond state pension age (SPA) and the extent to which the health consequences of pensioners are conditioned by whether the decision to retire or continue working is voluntary or involuntary. This study assessed the effect of work status beyond SPA on the long-term trajectories of cognitive and mental health for men and women separately, and the extent to which this relationship is conditioned by whether or not the choice to retire or continue working is voluntary or involuntary.

Methods Data are pensioners (aged between SPA and SPA+9) from the English Longitudinal Study of Ageing waves 4 (2008/09) through 9 (2018/2019). The analytic sample includes 1064 men and 1302 women for analyzing cognitive outcomes and 1167 men and 1404 women for analyzing depression. Growth curve models were applied.

Results Findings reveal that women who retired for involuntary reasons report a more precipitous decline in memory over time (coefficient for slope $=-0.070$; 95\%CI: -0.137 , $0.003)$. Involuntary retirement is psychologically distressing for men (baseline OR $=3.5$ ), with time this distress lessens (OR for slope $=0.883 ; 95 \% \mathrm{CI}$ : 0.781, 0.998); and, voluntary retirement, while not carrying any immediate benefit, translates into lower chances of developing depression over time for men (OR for slope $=0.796$; 95\%CI: 0.661, 0.959). Lastly, women who stay in work past SPA voluntarily are less likely to report depression at baseline $(\mathrm{OR}=0.331 ; 95 \% \mathrm{CI}$ : $0.158,0.693)$ and the differences in depression across different groups remain the same over time.

Discussion Our findings suggest that health in later life hinges less on whether a person is retired on time or working past SPA and more on the choice surrounding the decision to retire or continue working. Also, the health effect of retirement or extended work life is far from static; in fact, our findings suggest that it is more likely to shift over time. The complexity surrounding retirement demands that we continue to assess the impact of this transition on health within the context of individual characteristics, gender being one of them. Policies that extend working life should consider offering older people more personal control over decision surrounding retirement.

\section{OP30 DOES HIGH POLYGENIC PROPENSITY FOR ADHD PREDICT DIFFICULTIES IN LATE LIFE? EVIDENCE FROM THE ENGLISH LONGITUDINAL STUDY OF AGEING}

\footnotetext{
1,20lesya Ajnakina*, ${ }^{3,4,5}$ Theresa Wimberley, ${ }^{3,4,5}$ Søren Dalsgaard, ${ }^{6}$ Robin Murray, ${ }^{1}$ Andrew Steptoe. 'Behavioural Science and Health, University College London, London, UK; ${ }^{2}$ Biostatistics and Health Informatics, King's College London, London, UK; ${ }^{3}$ Economics and Business, School of Business and Social Sciences, Aarhus University, Aarhus, Denmark; ${ }^{4}$ Lundbeck Foundation Initiative for Integrative Psychiatric Research, Aarhus University, Aarhus, Denmark; ${ }^{5}$ Centre for Integrated Register-based Research (CIRRAU), Aarhus University, Aarhus, Denmark; ${ }^{6}$ Psychosis Studies, King's College London, London, UK
}

Background Attention-deficit/hyperactivity disorder (ADHD) can be viewed as the extreme end of heritable traits distributed in the general population. Due to the focus on children in the literature on $\mathrm{ADHD}$ and data constraints, to-date, almost no research focused on the relationship between ADHD-related outcomes in older adults from the general population. As ADHD is a highly heritable disorder, high genetic liability for ADHD may influence several important live domains in older adults; however, this question has not been investigated yet.

Methods Using a population representative sample of 6332 individuals aged $\geq 50$ with an average age 64.7 years (standard deviation $(\mathrm{SD})=9.3$, range $=50-101$ ) from the English Longitudinal Study of Ageing, we investigated relationships of genetic propensity to ADHD, as measured using polygenic score (PGS-ADHD), with several important life domains, such as occupational and social function, poor health, intimate relationships and cognition, which was measured employing tests for verbal memory and executive function. Information on physical health and socio-economic circumstances were selfreported by the participants; data on sexual activity and function were collected via the Sexual Relationships and Activities Questionnaire.

Results Compared to participants with low PGS-ADHD, older adults with high polygenic predisposition to ADHD were at a greater risk of lower educational attainment $(\mathrm{OR}=1.29$, $95 \% \mathrm{CI}=1.29-1.68, \mathrm{p}<0.001)$ and low accumulated wealth $(\mathrm{OR}=1.43, \quad 95 \% \mathrm{CI}=1.25-1.62, \quad \mathrm{p}<0.00)$; similar findings were observed in relation to lower verbal fluency score (Coef $=-0.62, \quad 95 \% \mathrm{CI}=-1.0-\quad-0.24, \quad \mathrm{p}<0.001)$ and verbal memory score $(\mathrm{Coef}=-0.37,95 \% \mathrm{CI}=-0.56--0.19, \mathrm{p}=0.001)$. Older adults with high PGS-ADHD had a significantly higher likelihood to report poor self-rated health $(\mathrm{OR}=1.42,95 \%$ $\mathrm{CI}=1.22-1.64, \mathrm{p}<0.001)$, body mass index $\geq 30 \quad(\mathrm{OR}=1.29$, $95 \% \mathrm{CI}=1.13-1.48, \mathrm{p}<0.001)$, and the presence of moderate to severe pain $(\mathrm{OR}=1.42,95 \% \mathrm{CI}=1.23-1.63, \mathrm{p}<0.001)$. They also had $36 \%$ higher odds of reporting a low or infrequent sexual activity in the past year $(95 \% \mathrm{CI}=1.16-1.60$, $\mathrm{p}<0.001)$.

Conclusion This is the first study to show that having a higher polygenic predisposition for ADHD is associated with poorer health, low socioeconomic status, limited sexual activity, and lower cognitive performance in older adults. These results highlight high polygenic risk for ADHD as an important risk factor poor functioning in several important life domains including poor health.

\section{Friday 17 September}

\section{Session: Covid Data \& Inequalities, $09.00-11.30$}

\section{OP31 A SNAPSHOT OF THE CHARACTERISTICS, QUALITY AND VOLUME OF THE COVID-19 EVIDENCE SYNTHESIS INFODEMIC: SYSTEMATIC REVIEW*}

'Jo Thompson Coon*, ${ }^{1}$ Rebecca Abbott, ${ }^{1}$ Alison Bethel, ${ }^{1}$ Morwenna Rogers ${ }^{1}$ Rebecca Whear, ${ }^{2}$ Liz Shaw, ${ }^{2}$ Noreen Orr, ${ }^{1}$ Ken Stein. ${ }^{1}$ NIHR Applied Research Collaboration, University of Exeter Medical School, University of Exeter, Exeter, UK; ${ }^{2}$ University of Exeter Medical School, University of Exeter, Exeter, UK 
Background Since the emergence of COVID-19, the academic and scientific community has reacted at pace to understand its epidemiology, diagnosis, treatment, prevention and impact. There are concerns that in the panic to get answers to help manage the pandemic, many of the cornerstones of robust methods are being omitted. The aim of this review was to map the nature, scope and quality of evidence syntheses on COVID-19 and to explore the relationship between review quality and the extent of researcher, policy and media interest.

Methods We conducted a systematic review of systematic reviews, rapid reviews, overviews and qualitative evidence syntheses addressing a research question relating to COVID-19. Searches were conducted in PubMed, Epistemonikos COVID19 evidence, the Cochrane Library of Systematic Reviews, The Cochrane COVID-19 Study Register, EMBASE, CINAHL, Web of Science Core Collection, and the WHO COVID-19 database in June 2020. Abstract and full text screening were undertaken by two independent reviewers. Descriptive information on review type, purpose, population, size, citation and attention metrics were extracted along with whether the review met six key methodological criteria. For reviews meeting all six methodological criteria, additional data were extracted on methods and publication metrics and AMSTAR-2 was used to assess the quality of the reported methods. Registration: PROSPERO CRD42020188822

Results Searches returned 2334 unique records. After applying eligibility criteria we included 280 reviews. Less than half reported undertaking critical appraisal and a third had no reproducible search strategy. There was considerable overlap in topics, with discordant findings. Eighty-eight of the 280 reviews met all six methodological criteria. Of these, 3 were rated as of moderate or high quality on AMSTAR-2, with the majority having critical flaws: only a third reported registering a protocol, and less than one in five searched named COVID19 databases. Review conduct and publication was rapid, with 56 of the 88 systematic review reported as being conducted within three weeks, and half published within three weeks of submission. Despite being of low quality and many lacking robust methods, the reviews received substantial attention across both academic and public platforms, and the attention was not related to the quality of review methods.

Conclusion Methodological flaws limit the validity of systematic reviews and the generalisability of their findings. Yet by being reported as 'systematic reviews', many readers may well regard them as high quality evidence, irrespective of the methods undertaken. To maintain trustworthiness, researchers, peerreviewers and journal editors need to ensure systematic reviews adhere to guidelines of best practice.

\section{OP32 JOB LOSS AND LOWER HEALTHCARE UTILIZATION DUE TO COVID-19 AMONG OLDER ADULTS ACROSS 27 EUROPEAN COUNTRIES}

${ }^{1}$ Gabriela Ksinan Jiskrova*, 1,2 Martin Bobák, 1,2Hynek Pikhart, 'Albert J Ksinan. ${ }^{1}$ RECETOX Faculty of Science, Masaryk University, Brno, Czech Republic; ${ }^{2}$ Department of Epidemiology and Public Health, University College London, London, UK

\subsection{6/jech-2021-SSMabstracts.32}

Background Older adults are one of the population groups at the highest risk of severe illness from COVID-19. However, little is known about the impact of the pandemic on economic activity and healthcare utilization for reasons unrelated to
COVID-19 among older adults. This study aimed to examine the prevalence and predictors of unemployment due to COVID-19 and healthcare utilization during the pandemic in a sample of older adults across 27 European countries. We examined the associations between individual and household demographic characteristics as well as country-level characteristics and the likelihood of the outcomes.

Methods We utilized cross-sectional data from the large multinational Study of Health, Ageing and Retirement in Europe (SHARE) COVID-19 Survey, collected between June and August 2020. All participants $(n=52,061)$ reported whether they forwent medical treatment and whether their appointment was postponed due to COVID-19. Economically active participants $(\mathrm{n}=10,958)$ reported whether they lost a job due to COVID-19. Three-level hierarchical models were estimated for each outcome to test the effects of individual, household, and country-level characteristics.

Results The mean prevalence of reported job loss, forgone, and postponed medical care were 19\%, 12\%, and 26\%, respectively. Women we more likely to lose their job than men (OR 1.27; 95\% CI 1.14-1.41 at mean age) and this effect was larger for older women. Covid-related job loss was also associated with household income (OR per 1,000 EUR was 0.84 ; 95\% CI $0.78-0.90$ ) and lower education (OR comparing primary vs. tertiary education was 1.27 ; 95\% CI 1.14 1.41). Forgone and postponed medical care was associated with older age in men, female sex, and higher education. For example, women were more likely to forgo medical treatment compared to men (OR 1.63; 95\% CI 1.54-1.73 at mean age). At the country level, postponed medical care was associated with more stringent governmental anti-COVID measures (OR for inter-quartile range of stringency index was $1.48 ; 95 \% \mathrm{CI}$ 1.14-1.93).

Conclusion Job loss and lower healthcare utilization for nonCOVID-19 related reasons were prevalent among older adults in the SHARE sample and were associated with several sociodemographic and country characteristics. Job loss appeared to disproportionally affect already economically vulnerable individuals, which may contribute to an exacerbation of social inequalities over time. Additionally, the results highlighted the importance of focusing on maintaining access to healthcare during the lockdown and following up on any missed medical appointments to prevent increased morbidity due to missed screenings and treatment.

\section{OP33 DIGITAL EXCLUSION DURING THE COVID-19 PANDEMIC IN THE ENGLISH LONGITUDINAL STUDY FOR AGEING POPULATION}

Shivan Thakrar*, Jennifer Cole, Helen Parretti, Nicholas Steel. Public Health and Health Services Research, University of East Anglia, Norwich, Norfolk, UK

\subsection{6/jech-2021-SSMabstracts.33}

Background The NHS long term plan commits to 'digital first primary care' by 2024. Increasing reliance on digital access may disadvantage those who do not use the internet. We aimed to assess changes in internet use in adults over 50 years of age before and during the coronavirus pandemic.

Methods Participants in the English Longitudinal Study for Ageing were asked how often they used the internet or email in Wave 9 (W9) from June 2018 to June 2019 and COVID Wave 1 (CW1) from June to July 2020. Response options 